\title{
Bioavailability of procyanidin dimers and trimers and matrix food effects in in vitro and in vivo models
}

\author{
Aida Serra ${ }^{1}$, Alba Macià ${ }^{1}$, Maria-Paz Romero ${ }^{1}$, Josep Valls ${ }^{2}$, Cinta Bladé ${ }^{3}$, Lluís Arola ${ }^{3}$ \\ and Maria-José Motilva ${ }^{1 *}$ \\ ${ }^{1}$ Department of Food Technology, XeRTA-UTPV, Escola Tècnica Superior d'Enginyeria Agrària, Universitat de Lleida, \\ Avda/Alcalde Rovira Roure 191, 25198 Lleida, Spain \\ ${ }^{2}$ Shirota Functional Foods, S.L., Reus, Spain \\ ${ }^{3}$ Department of Biochemistry and Biotechnology, XeRTA-UE, Universitat Rovira i Virgili, C/Marcel-li Domingo s/n, 43007 \\ Tarragona, Spain
}

(Received 22 July 2009 - Revised 6 October 2009 - Accepted 8 October 2009 - First published online 14 December 2009)

\begin{abstract}
Among procyanidins (PC), monomers, such as catechin and epicatechin, have been widely studied, whereas dimer and trimer oligomers have received much less attention, despite their abundance in our diet. Recent studies have showed that as dimers and trimers could be important in determining the biological effects of procyanidin-rich food, understanding their bioavailability and metabolism is fundamental. The purpose of the present work is to study the stability of PC under digestion conditions, the metabolism and the bioavailability by using a combination of in vitro and in vivo models. Simultaneously, the matrix effect of a carbohydrate-rich food on the digestibility and bioavailability of PC is investigated. The results show a high level of stability of PC under gastric and duodenal digestion conditions. However, the pharmacokinetic study revealed limited absorption. Free forms of dimers and trimers have been detected in rat plasma, reaching the maximum concentration $1 \mathrm{~h}$ after oral intake of a grape seed extract.
\end{abstract}

Procyanidins: Dimers: Trimers: Digestion: Bioavailability

Procyanidins (PC) are found in most plants and in a wide range of foods, such as red wine, cocoa, tea and fruits, and thus they are a part of the human diet. PC belong to the group of flavonoids, and are phenolic compounds mainly formed of $(+)$-catechin and (-)-epicatechin units with $\mathrm{C}_{4}-\mathrm{C}_{8}$ and/or $\mathrm{C}_{4}-\mathrm{C}_{6}$ bonds. $\mathrm{PC}$ are formed from the condensation of monomeric units, between two and five units for oligomers and over five units for polymers ${ }^{(1)}$.

Grape seed procyanidin extracts (GSPE) have shown several bioactivities. They improve antioxidant cell defences ${ }^{(2)}$ and the plasma lipid profile ${ }^{(3,4)}$, limit adipogenesis ${ }^{(5)}$ and function as insulinomimetic ${ }^{(6)}$ and anti-inflammatory ${ }^{(7)}$ agents. A preliminary study of an in vitro model by the authors has also determined that dimeric and trimeric oligomers are the most powerful PC molecules that mimic the complete $\mathrm{GSPE}^{(8)}$. Thus, to explain these health effects of PC and understand the mechanism by which $\mathrm{PC}$ act at the cellular level in vivo, it is essential to determine $\mathrm{PC}$ stability during the digestive process as well as their bioavailability.

Despite it being clear that monomers are absorbed in human subjects and animals, there are controversies about the bioavailability of oligomeric forms. Different studies, following the ingestion of chocolate ${ }^{(9-12)}$, black and green tea ${ }^{(13-16)}$, red wine ${ }^{(17,18)}$ and grape seed extract ${ }^{(1)}$, have shown that during digestion, the oligomers are fragmented into monomeric units of catechin and epicatechin. These are then absorbed, appearing in plasma and urine primarily as glucuronidated, methylated and sulphated metabolites. A study by Rios et al. ${ }^{(19)}$ with six healthy subjects who consumed a rich PC cocoa beverage studied the depolymerisation of PC in the stomach and proved that they were remarkably stable in the stomach environment. Another study by Sano et al. ${ }^{(20)}$ was the first work to detect procyanidin B1 in human serum after oral intake of a GSPE, the maximum concentration appearing $2 \mathrm{~h}$ after intake. As well as stability during digestion, the dose of intake could determine the primary site of phenol metabolism. Large doses are metabolised primarily in the liver, and small doses may be metabolised by the intestinal mucosa, with the liver playing a secondary role to further modify the polyphenol conjugates from the small intestine ${ }^{(21)}$.

The bioavailability of polyphenols has been also reviewed, especially to focus on intestinal absorption and the influence of their chemical structure (for example glycosylation, esterification and polymerisation) and the effect of the food matrix composition $^{(22)}$. Different studies have been carried out into the interaction between polyphenols and a food matrix such as milk ${ }^{(23,24)}$, olive oil ${ }^{(25)}$ or sugar ${ }^{(26)}$. The evidence suggests that variations in polyphenol absorption also occur due to

Abbreviations: GSPE, grape seed procyanidin extract; IN, fraction found inside dialysis solution; OUT, fraction found outside dialysis solution; PC, procyanidins. *Corresponding author: Dr Maria-José Motilva, fax +34 973 702596, email motilva@tecal.udl.es 
interactions between polyphenols and other food components. For example, some authors suggest that the carbohydrate composition of the meal may influence flavonoid absorption through an effect on the motility of gastric secretions and hepatic blood flow ${ }^{(26)}$. The polyphenol-protein interaction has also been studied, and shown to be similar to antigenantibody interactions. The results of these studies suggest that interactions may occur between digestive proteins and monomers and dimers of flavanols from grape seed extract during pancreatic digestion ${ }^{(27)}$.

Nevertheless, one of the most important limitations of the bioavailability studies in human subjects or animal models is related to inter-individual variability. In vitro digestion models are a very useful tool for studying the stability of food components and evaluating the potential effects of the food matrix on their bioavailability. Reviewing the literature, a wide range of in vitro digestion methods have been designed to simulate the effect of digestion on food or food components and study its consequences. In vitro digestion methods can be classified into two categories ${ }^{(28)}$ : static $^{(29)}$; dynamic ${ }^{(30,31)}$ gastrointestinal models. The static models simulate the transit of alimentary bolus through the human digestive tract by sequential exposure of food to simulated mouth, gastric and small intestinal conditions. In contrast, the dynamic gastrointestinal models mimic the gradual transit of ingested compounds through the digestive tract.

Other important limitation related to the study of PC bioavailability concerns the low concentration of their metabolites in biological samples ${ }^{(1)}$. Therefore, measuring them requires a precise, robust, sensitive and selective analytical technique that allows them to be identified and quantified. Liquid chromatography with MS is a powerful tool for both confirmatory and quantitative analyses in complex matrices due to its high sensitivity and selectivity. Apart from the analytical technique, the use of an adequate extraction procedure to minimise the matrix effects is very important.

As PC dimers and trimers could be important in determining the biological effects of PC-rich food, understanding the bioavailability and metabolism of PC oligomers is fundamental. The objective of the present research is to study the stability of PC under digestion conditions, the metabolism and the bioavailability by using a combination of in vitro and in vivo models. Simultaneously, the matrix effect of a carbohydraterich food on digestibility and bioavailability of PC is investigated.

\section{Experimental methods}

\section{Chemicals and reagent}

A GSPE was used as the source of PC. The PC content of the extract was $>75 \%$ (monomers $22 \%$, dimers $20 \%$ and trimers to pentamers $56 \%$ ). The composition of commercial cerealbased food, used as carbohydrate-rich food, was as follows: starch (hydrolysed wheat, wheat, barley, maize and oats); malt extract; mineral salts ( $\mathrm{Ca}, \mathrm{Fe}$ and $\mathrm{Zn})$; vitamins $(\mathrm{C}$, niacin, $\mathrm{E}$, thiamine, $\mathrm{A}, \mathrm{B}_{6}, \mathrm{~B}_{9}$ and $\left.\mathrm{D}\right)$; flavouring (vanillin).

Internal standard catechol and the standards of (-)-epicatechin and $(+)$-catechin were purchased from Sigma Aldrich (St Louis, MO, USA); procyanidin B2 from Fluka Co. (Buchs, Switzerland) and trimer was isolated from phenolic extract obtained from cocoa nibs ${ }^{(32)}$ using the technique described by Serra et al. ${ }^{(33)}$. The cocoa nibs were kindly donated by La Morella Nuts, S.A. (Reus, Spain). Individual stock standard solutions and the internal standard were dissolved in methanol and stored in a dark-glass flask at $-18^{\circ} \mathrm{C}$. Standard mixtures, at different concentrations, were prepared by appropriate dilution of the stock solutions, and these were stored in dark-glass flasks at $4^{\circ} \mathrm{C}$ before chromatographic analysis.

Acetonitrile (HPLC grade), methanol (HPLC grade), acetone (HPLC grade) and glacial acetic acid $(\geq 99.8 \%$ ) were of analytical grade (Scharlab, Barcelona, Spain). Ultrapure water was obtained from a Milli-Q water purification system (Millipore Corporation, Bedford, MA, USA).

\section{Simulated gastrointestinal digestion}

A gastrointestinal in vitro digestion model was performed based on the methodology described by Ortega et al. ${ }^{(34)}$. The discontinuous model describes a three-step procedure to mimic the digestive process in the mouth, stomach (gastric digestion) and small intestine (duodenal digestion) (Fig. 1). In order to evaluate the food matrix effect, two different samples were subjected to the digestion model: $300 \mathrm{mg}$ PC extract (GSPE); $300 \mathrm{mg}$ GSPE + 600 mg carbohydrate-rich food. After the gastric step, the digestion mixture was placed in tubes and centrifuged for $15 \mathrm{~min}$ at $12000 \mathrm{~g}$ at $4^{\circ} \mathrm{C}$, yielding the chyme (supernatant or soluble fraction) and the pellet (cloud or non-soluble fraction) ${ }^{(34)}$. Both fractions, the chyme and pellet, were freeze-dried (BMG Labtech,
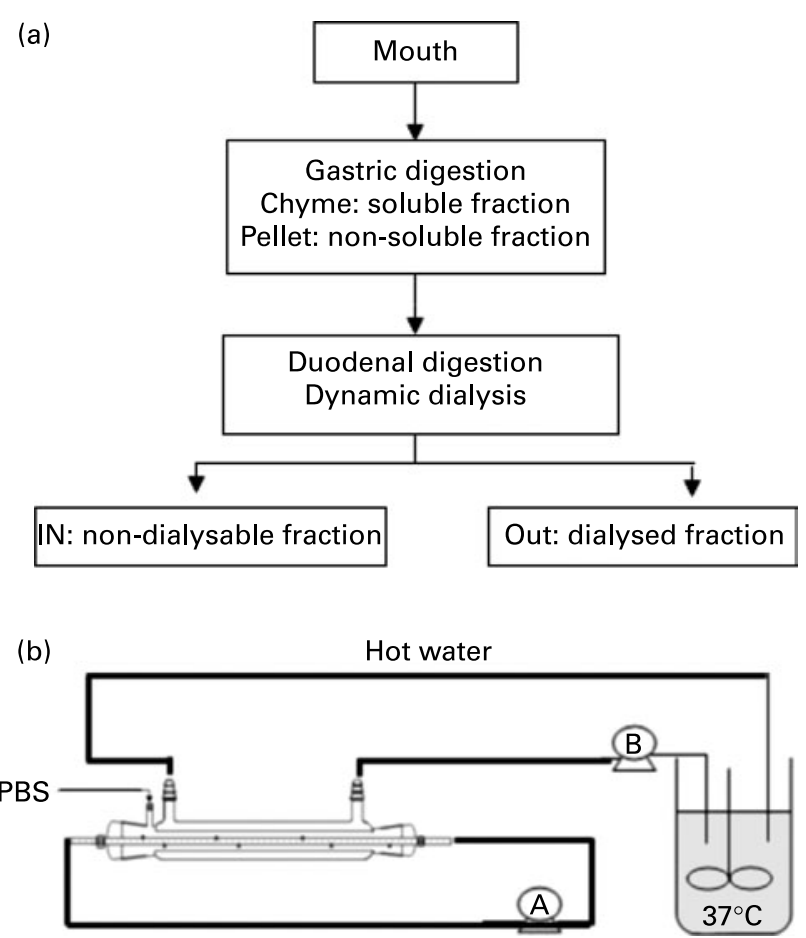

$\mathrm{DD}(1 \mathrm{ml} / \mathrm{min})$

Fig. 1. (a) Steps of the proposed in vitro digestion model. (b) Schema of the duodenal digestion with dynamic dialysis. DD, duodenal mixture (fraction found inside dialysis solution (IN)); PBS (fraction found outside dialysis solution (OUT)). 
Offenburg, Germany) and stored for chromatographic analysis of the PC content.

At the end of the dialysed duodenal digestion step, two fractions were collected and analysed separately ${ }^{(34)}$ : the outside dialysis solution (OUT) (Fig. 1), which was considered the dialysable fraction; and the inside dialysis tub content (IN), referring to the non-dialysable fraction. The dialysable fraction (OUT) is considered the fraction that could be available for absorption into the systematic circulation by passive diffusion; while the non-dialysable fraction (IN) is attributed to the digested fraction that would reach the colon fermentation intact. Both fractions were freeze-dried and stored for chromatographic analysis of the PC content.

\section{Chromatographic analysis of procyanidins in digested fractions}

The digested freeze-dried fractions (chyme, pellet, IN and OUT) were dissolved in acetone-Milli-Q water-acetic acid $(70: 29 \cdot 5: 0 \cdot 5, \mathrm{v} / \mathrm{v} / \mathrm{v})$ at a ratio of 1:5. The tubes were vortexed for $5 \mathrm{~min}$ and centrifuged at $12500 \mathrm{rpm}$. The supernatant containing the solubilised phenols was filtered through $0.22 \mu \mathrm{m}$ nylon filters before chromatographic analysis by ultraperformance liquid chromatography-MS/MS using a Waters Acquity ultraperformance liquid chromatography system (Waters, Milford, MA, USA), equipped with a binary pump system (Waters) ${ }^{(32)}$. The ultraperformance liquid chromatography analyses were performed using an Acquity high strength silica T3 column $(100 \mathrm{~mm} \times 2.1 \mathrm{~mm}$ inner diameter, $1.8 \mu \mathrm{m}$ particle size; Waters) with a binary mobile phase. Solvent A was water-acetic acid (0.2\%), and the solvent as acetonitrile. Catechin and epicatechin were quantified using the curves of the respective standards. Epigallocatechin, epicatechin gallate and epigallocatechin gallate were quantified using the epicatechin curve. The PC, dimers and trimers were quantified with the calibration curves of the respective standards. The oligomers from tetramer to heptamer were quantified with the trimer calibration curve. The results of the quantification of the PC in pellet (non-soluble fraction) are expressed as $\mu$ mol per $g$ of pellet, and the results of the quantification of the PC in the chyme (soluble fraction), IN and OUT fractions are expressed in $\mu \mathrm{M}$.

\section{Pharmacokinetic study}

Rat plasma samples were obtained from 3-month-old male Wistar rats weighing between 370 and $420 \mathrm{~g}$ and were purchased from Charles River Laboratories (Barcelona, Spain). The Animal Ethics Committee of the Rovira i Virgili University approved all the procedures. The rats were housed in temperature-controlled rooms $\left(22^{\circ} \mathrm{C}\right)$ and subjected to a standard $12 \mathrm{~h}$ light $-12 \mathrm{~h}$ dark cycle (with a light period of 09.00 21.00 hours). All the animals were fed a standard diet of PanLab A04 (Panlab, Barcelona, Spain) and water.

After the animals had been in fasting conditions for between 16.00 and 17.00 hours with only access to tap water, two experiments were carried out: a dose of the PCrich extract, $1 \mathrm{~g}$ GSPE per $\mathrm{kg}$ of body weight dissolved in water; a dose of $2 \mathrm{~g}$ carbohydrate-rich food $+1 \mathrm{~g}$ GSPE for $\mathrm{kg}$ of body weight dissolved in water, which was administered to the rats by intragastric gavage. Three animals per experiment were terminally anaesthetised with ketaminexylazine and euthanised by exsanguinations at $0 \mathrm{~h}$ (fasting conditions), 1, 2 and $4 \mathrm{~h}$ (postprandial conditions). Blood samples were collected from the abdominal aorta with heparin-moistened syringes. The plasma samples were obtained by centrifugation $\left(2000 \mathrm{~g}, 30 \mathrm{~min}\right.$ at $\left.4^{\circ} \mathrm{C}\right)$ and stored at $-80^{\circ} \mathrm{C}$ until the chromatographic analysis of the PC. The identification of the PC metabolites was performed by analyses in MS (full-scan mode) and MS/MS (based on neutral loss scan and product ion scan mode) $)^{(33)}$. First, analyses were carried out in the full-scan mode (from 419 to $1200 \mathrm{~m} / \mathrm{z}$ ) by applying different cone voltages from 20 to $60 \mathrm{~V}$. When low cone voltages were applied, the MS spectrum gave information about the precursor ion or the $[\mathrm{M}-\mathrm{H}]^{-}$. In contrast, when high cone voltages were applied, specific fragment ions were generated and the MS spectrum gave information about their structure. The structural information was also verified by using product ion scan and neutral loss scan in the MS/MS mode.

All the samples were analysed in triplicate by ultraperformance liquid chromatography-MS/MS according to the method in Serra et al. ${ }^{(33)}$. The quantification of the free forms of the PC and their metabolites in the plasma samples was done using the calibration curves of the respective free forms, and the concentrations were expressed as catechin or epicatechin equivalents.

\section{Statistical analysis}

All the data are expressed as the mean of three replicates. In order to simplify the results shown in the tables, we omitted the standard deviation because all these values were lower than $10 \%$. The data were analysed by a one-way ANOVA test to assess the effect of the carbohydrate-rich food on the in vitro digestibility and in vivo pharmacokinetics of $\mathrm{PC}$ from the GSPE extract. A significant difference is considered at a level of $P<0 \cdot 05$. All the statistical analysis was carried out using STATGRAPHICS Plus 5.1.

\section{Results}

Evaluation of the stability of procyanidins in digestion by the in vitro simulated digestion model

The first part of the study was focused on the evaluation of the stability of PC during digestion using an in vitro model. The composition of the GSPE used as a PC and proanthocyanidin source in the study is summarised in Table 1. Dimers and trimers were the most abundant compounds with 250.18 and $1568.72 \mu \mathrm{mol} / \mathrm{g}$ extract, respectively. The digestion procedure was applied to GSPE dissolved in water, and the same extract was mixed with cereal-based food in order to evaluate the influence of the food matrix with a high-carbohydrate content on the digestion stability of the PC.

The compounds determined initially in GSPE (Table 1) were analysed in all fractions of in vitro digestions to evaluate their digestibility and stability. After the gastric step, the content of PC and proanthocyanidins was measured in the soluble and non-soluble fractions, respectively, called the chyme and pellet (Fig. 1), and the results were expressed as $\mu$ mol of concentration in the chyme fraction and as $\mu \mathrm{mol}$ per $\mathrm{g}$ of 
Table 1. Phenolic composition of grape seed procyanidin extract $($ GSPE)*

\begin{tabular}{lc}
\hline Compound & Concentration $(\mu \mathrm{mol} / \mathrm{g})$ \\
\hline Catechin & 58 \\
Epicatechin & 52 \\
Epigallocatechin & $5 \cdot 50$ \\
Epicatechin gallate & 89 \\
Epigallocatechin gallate & $1 \cdot 40$ \\
Dimer & 250 \\
Trimer & 1568 \\
Tetramer & $8 \cdot 8$ \\
Pentamer & 0.73 \\
Hexamer & 0.38 \\
Heptamer & 0 \\
\hline
\end{tabular}

${ }^{*}$ The results are expressed as $\mu \mathrm{mol} / \mathrm{g}$ of GSPE.

pellet (Table 2). After the gastric step, the amount of PC in the chyme was higher than in the pellet fraction independently of the presence of the carbohydrate-rich food in the digestion mixture. The PC oligomers, dimers to heptamers, showed higher concentrations than the monomers (catechin and epicatechin) in the chime and pellet fractions, the trimer form being the most abundant compound in the gastric fractions, reaching a concentration of $50279.77 \mu \mathrm{mol}$ in the chyme fraction after the gastric step. Nevertheless, forms with a high degree of polymerisation (pentamer, hexamer and heptamer) were not detected in pellet fraction.

After the duodenal step, an important loss of PC was observed, and most were retained in the non-dialysable fraction (IN). Nevertheless, dimers and trimers were quantified in the dialysable (OUT) fraction (Fig. 1), as well as the monomeric structures. As a consequence of the duodenal digestion conditions, decomposition of oligomeric forms was observed in the respective monomeric forms. Additionally to the depolymerisation of GSPE, a decrease in the ratio catechin/epicatechin was observed in the digestion mixture. Initially, in the GSPE extract, this ratio was approximately $1 \cdot 1$ and after digestion, a change in the ratio of roughly 0.7 was observed in all fractions.
Finally, the comparative analysis between the concentrations of the different $\mathrm{PC}$ in the digestion mixtures revealed that the presence of carbohydrate-rich food, as a source of carbohydrates, showed a significant effect on the digestibility of PC in the in vitro digestion model. When PC and proanthocyanidins were submitted to digestion together with carbohydrate-rich food, the amount of catechin and epicatechin present in the digestion mixture after the gastric step was significantly higher $(P<0 \cdot 05)$ (Table 2$)$. By contrast, the concentration of the other compounds was greater when GSPE was digested without carbohydrate-rich food. Greater differences were observed in the non-soluble fraction (pellet) (Fig. 1), showing a higher concentration of PC $(P<0.05)$ when the extract was digested with carbohydrate-rich food. By contrast, the effect of carbohydrates on the digestibility during duodenal digestion was lower. Thus, the concentration of PC in the dialysed fraction (OUT) was similar independently of the presence of carbohydrates, with the exception of the epigallocatechin gallate that showed a lower concentration $(P<0.05)$ in the presence of carbohydrates. On the other hand, significant differences were observed in the non-dialysable fraction (IN), except the forms with a higher degree of polymerisation (pentamers to heptamers), which showed the same concentration under the two digestion conditions. However, no clear tendency was observed in the results of non-dialysable (IN) fraction in relation to the presence or absence of carbohydrates.

\section{Pharmacokinetic of procyanidins}

Figs. 2 and 3 show the results of the pharmacokinetic study of rat plasma after the ingestion of GSPE without and with carbohydrate-rich food. Under basal conditions (time $=0$ ), PC were not detected in rat plasma. Two hours after the GSPE ingestion, several metabolites, such as catechin glucuronide and epicatechin glucuronide, reached peak plasma concentrations with 6.24 and $9.74 \mathrm{nM}$, respectively, when GSPE was ingested with carbohydrate-rich food, and 6.32 and $8.71 \mathrm{nM}$ when GSPE is ingested without carbohydrate-rich food. In contrast, the concentration of PC, free forms of

Table 2. Procyanidin and proanthocyanidin contents in the different fractions of the gastric and duodenal steps of the simulated gastrointestinal digestion of grape seed procyanidin extract (GSPE) without and with carbohydrate-rich food

\begin{tabular}{|c|c|c|c|c|c|c|c|c|}
\hline \multirow[b]{3}{*}{ Compound } & \multicolumn{4}{|c|}{ GSPE } & \multicolumn{4}{|c|}{ GSPE + carbohydrate-rich food } \\
\hline & \multicolumn{2}{|c|}{ Gastric } & \multicolumn{2}{|c|}{ Duodenal } & \multicolumn{2}{|c|}{ Gastric } & \multicolumn{2}{|c|}{ Duodenal } \\
\hline & Chyme $(\mu \mathrm{M})$ & Pellet $(\mu \mathrm{mol} / \mathrm{g})$ & OUT $(\mu \mathrm{M})$ & IN $(\mu \mathrm{M})$ & Chyme $(\mu \mathrm{M})$ & Pellet $(\mu \mathrm{mol} / \mathrm{g})$ & OUT $(\mu \mathrm{M})$ & IN $(\mu \mathrm{M})$ \\
\hline Catechin & $1755^{\mathrm{a}}$ & $7 \cdot 4^{\mathrm{a}}$ & $95^{\mathrm{a}}$ & $451^{\mathrm{a}}$ & $1928^{b}$ & $37^{\mathrm{b}}$ & $83^{\mathrm{a}}$ & $608^{b}$ \\
\hline Epicatechin & $2221^{a}$ & $9 \cdot 6^{\mathrm{a}}$ & $126^{a}$ & $613^{\mathrm{a}}$ & $2499^{b}$ & $42^{\mathrm{b}}$ & $114^{\mathrm{a}}$ & $823^{\mathrm{b}}$ \\
\hline Epigallocatechin & $258^{\mathrm{a}}$ & $0.49^{\mathrm{a}}$ & $2 \cdot 6^{\mathrm{a}}$ & $37^{\mathrm{a}}$ & $186^{\mathrm{b}}$ & $1.41^{\mathrm{b}}$ & $2 \cdot 4^{\mathrm{a}}$ & $34.5^{\mathrm{b}}$ \\
\hline Epicatechin gallate & $6305^{\mathrm{a}}$ & $29 \cdot 8^{a}$ & $109^{\mathrm{a}}$ & $1772^{\mathrm{a}}$ & $5272^{b}$ & $191^{\mathrm{b}}$ & $72^{\mathrm{b}}$ & $2111^{\mathrm{b}}$ \\
\hline Epigallocatechin gallate & $136^{\mathrm{a}}$ & $0.409^{a}$ & $0.80^{\mathrm{a}}$ & $28 \cdot 7^{\mathrm{a}}$ & $91^{\mathrm{b}}$ & $1.9^{b}$ & $0.65^{a}$ & $25 \cdot 3^{\mathrm{b}}$ \\
\hline Dimer & $12953^{a}$ & $37 \cdot 1^{\mathrm{a}}$ & $197^{\mathrm{a}}$ & $3517^{\mathrm{a}}$ & $11485^{\mathrm{b}}$ & $124^{\mathrm{b}}$ & $168^{\mathrm{a}}$ & $4005^{\mathrm{b}}$ \\
\hline Trimer & $50279^{a}$ & $109^{a}$ & $147^{a}$ & $8101^{a}$ & $37505^{\mathrm{b}}$ & $356^{\mathrm{b}}$ & $111^{\mathrm{a}}$ & $8659^{a}$ \\
\hline Tetramer & $10076^{\mathrm{a}}$ & $21 \cdot 0^{\mathrm{a}}$ & $0.25^{\mathrm{a}}$ & $1748^{\mathrm{a}}$ & $5368^{\mathrm{b}}$ & $82^{\mathrm{b}}$ & ND & $1673^{\mathrm{b}}$ \\
\hline Pentamer & $16 \cdot 8^{\mathrm{a}}$ & ND & ND & $12 \cdot 7^{a}$ & ND & ND & ND & ND \\
\hline Hexamer & $185^{a}$ & ND & ND & $16 \cdot 9^{\mathrm{a}}$ & $49^{\mathrm{b}}$ & ND & ND & $6 \cdot 6^{\mathrm{a}}$ \\
\hline Heptamer & $0.66^{\mathrm{a}}$ & ND & ND & $0.73^{\mathrm{a}}$ & $2 \cdot 34^{\mathrm{b}}$ & ND & ND & ND \\
\hline
\end{tabular}

OUT, fraction found outside dialysis solution; IN, fraction found inside dialysis solution; ND, not detected.

${ }^{\mathrm{a}, \mathrm{b}}$ Mean values within a column with unlike superscript letters were significantly different $(P<0.05)$ between GSPE + carbohydrate-rich food and GSPE in each digestion step (unpaired Student's $t$ test). 

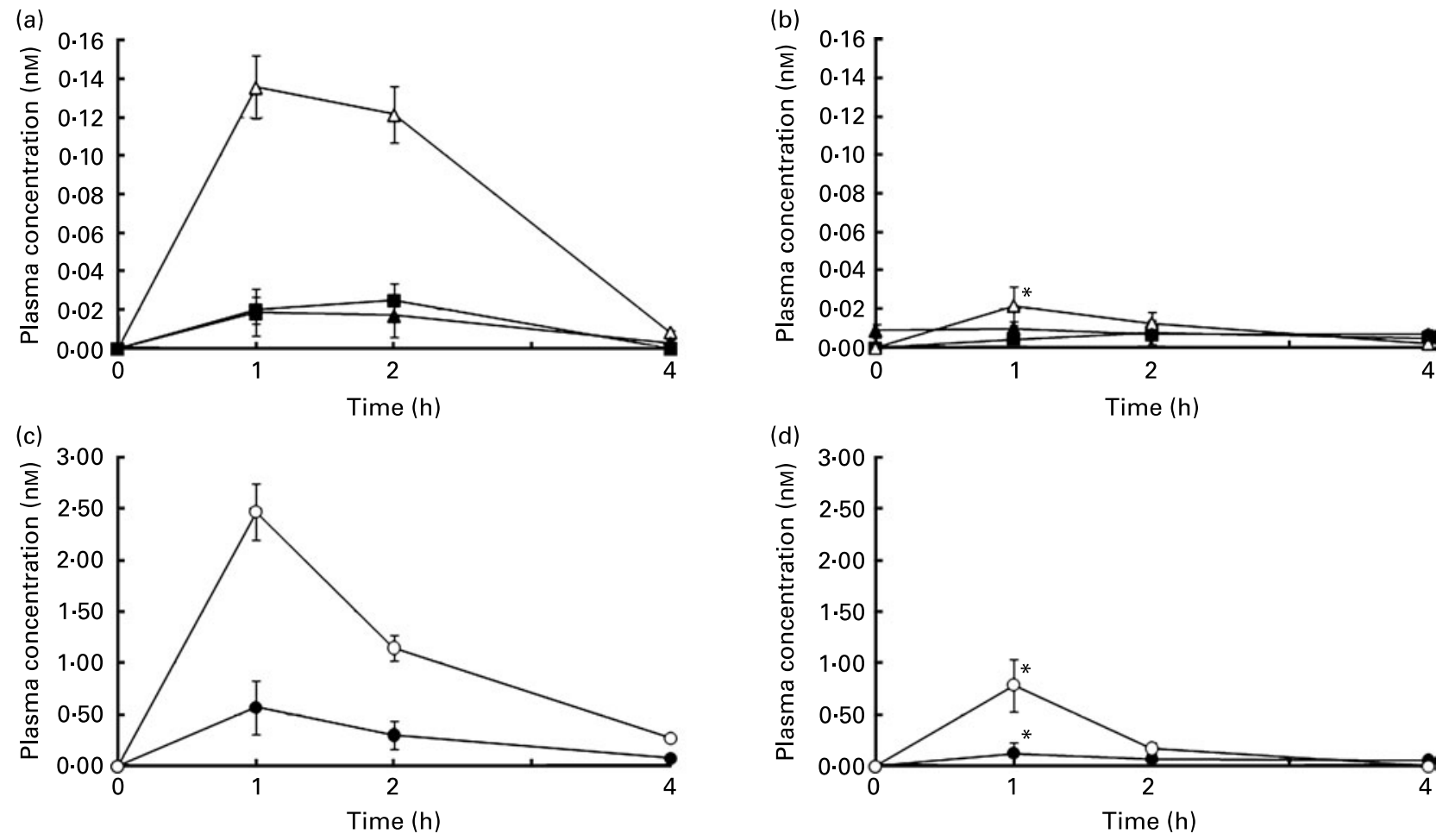

Fig. 2. Pharmacokinetic curves of monomeric forms, $(-\mathbf{-}-)$ catechin, $(-\Delta-)$ epicatechin $(-\mathbf{\Delta}-)$ and epicatechin gallate (a and b), and polymeric forms, $(-\bullet-)$ dimer and (-O-) trimer (c and d), detected in rat plasma collected between 0 and $4 \mathrm{~h}$ after the ingestion of grape seed procyanidin extract with and without carbohydrate-rich food. The results are expressed as nM. * Mean value was significantly different from that without carbohydrate-rich food $(P<0.05$; unpaired Student's $t$ test).
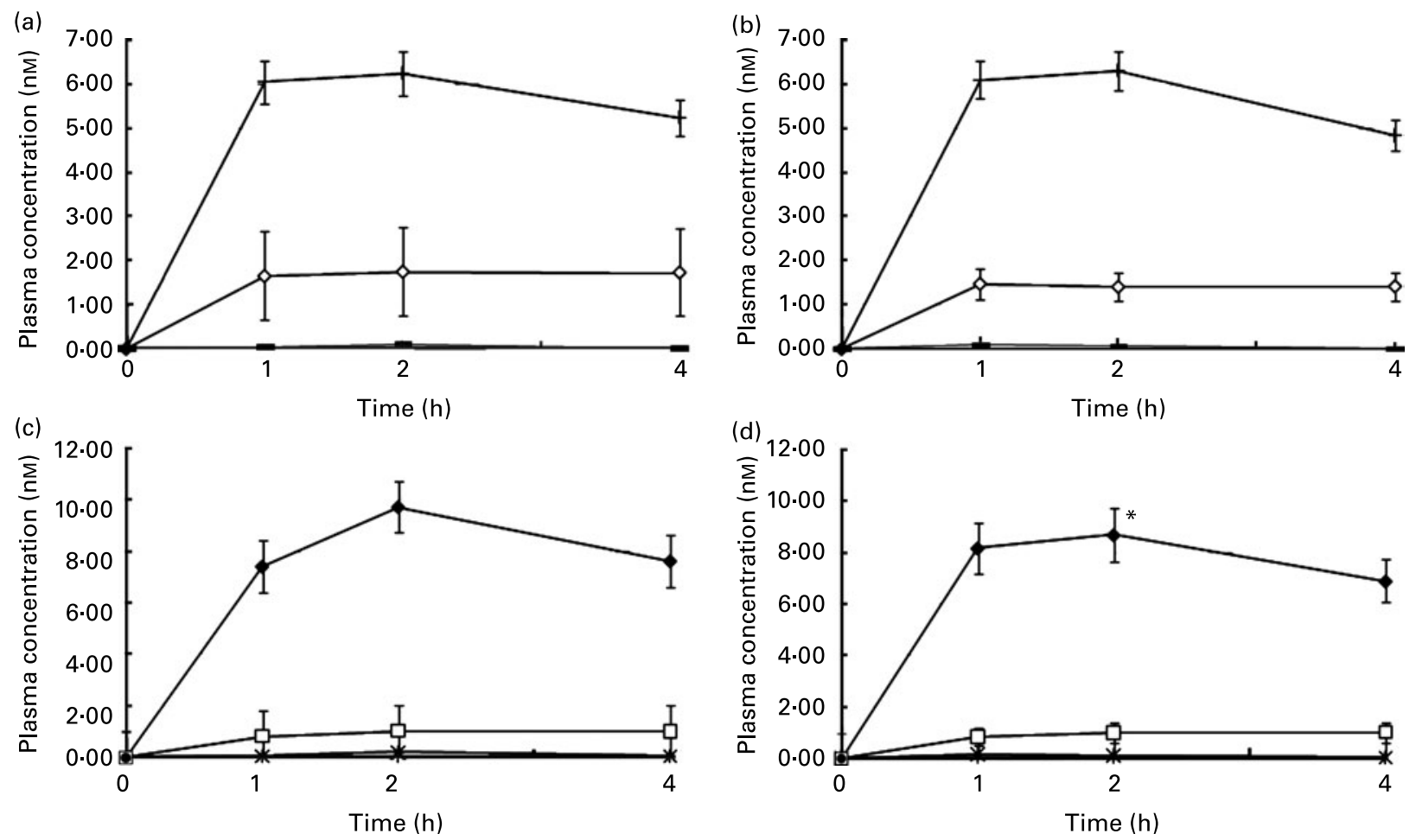

Fig. 3. Pharmacokinetic curves of grape seed procyanidin extract (GSPE) metabolites according to the monomer structure, being (- -$)$ catechin methyl glucuronide, $(-+-)$ catechin glucuronide and (- - ) catechin methyl sulphate $(a$ and $b)$, and $(-\square-)$ epicatechin methyl glucuronide, $(-\bullet-)$ epicatechin glucuronide and $(\rightarrow-)$ epicatechin methyl sulphate (c and d), detected in rat plasma collected between 0 and $4 \mathrm{~h}$ after the ingestion of GSPE with and without carbohydrate-rich food. The results are expressed as nM. ${ }^{*}$ Mean value was significantly different from that without carbohydrate-rich food $(P<0.05$; unpaired Student's $t$ test). 
dimers and trimers in the plasma reached a maximum $1 \mathrm{~h}$ after administration. These free forms appeared in the plasma faster than the glucuronidated forms of the monomers. Dimer concentration was greater after the ingestion of GSPE without the presence of carbohydrate-rich food, reaching a postprandial concentration in plasma of $0.57 \mathrm{nM}$ as against $0.12 \mathrm{~nm}$ when the GSPE was ingested with carbohydrate-rich food. Similarly, the presence of the carbohydrate-rich food reduced the maximum concentration of the trimer in the plasma. Nonconjugated forms of monomers (catechin, epicatechin and epicatechin gallate) were present in the plasma at very low concentration, less than $0.03 \mathrm{nM}$ under the two experimental conditions. Glucuronidated forms of catechin and epicatechin were the most abundant, and the second most abundant metabolite was methyl-glucuronidated forms, independently of the presence of the carbohydrate-rich meal.

Comparison between in vitro digestion method and in vivo bioavailability

Fig. 4(a) and (b) show the percentage of different PC present in the dialysed fraction (OUT) of the duodenal digestion mixture corresponding to the in vitro digestion model (Fig. 1); and Fig. 4(c) and (d) show the percentage of free PC and their metabolites in the rat plasma after the ingestion of the GSPE extract (in vivo model). The expression of the quantification of
$\mathrm{PC}$ as percentages in both the in vitro and in vivo models aims to normalise the results to facilitate their comparative analysis independently of whether the concentrations of the compounds varied widely in both models. In all the plasma samples, epicatechin was the most abundant compound, glucuronidated forms being 88 and $89 \%$ of the total, when GSPE was digested alone and with carbohydrate-rich food, respectively. This was followed by catechin, which was the second most abundant compound. Nevertheless, the dimer structure was the most substantial PC quantified in the dialysed fraction (OUT) after the duodenal step of the in vitro digestion model, reaching a percentage of almost 30\% (Fig. 4(a) and (b)); however, this compound was a very low percentage in the plasma samples (1.69\% when the GSPE was administered alone and $0.35 \%$ when it was administered with carbohydraterich food) (Fig. 4(c) and (d)). The quantification of the trimer in the plasma samples showed percentages under $1 \%$, independently of the presence or absence of carbohydrate-rich food in the intake, whereas its percentage in the dialysed fraction (OUT) of the duodenal digestion was higher, close to $20 \%$. Epicatechin gallate behaved similarly.

\section{Discussion}

We have shown, using in vitro cell lines, that dimer and trimer $\mathrm{PC}$ are the most powerful molecules that mimic the in vivo
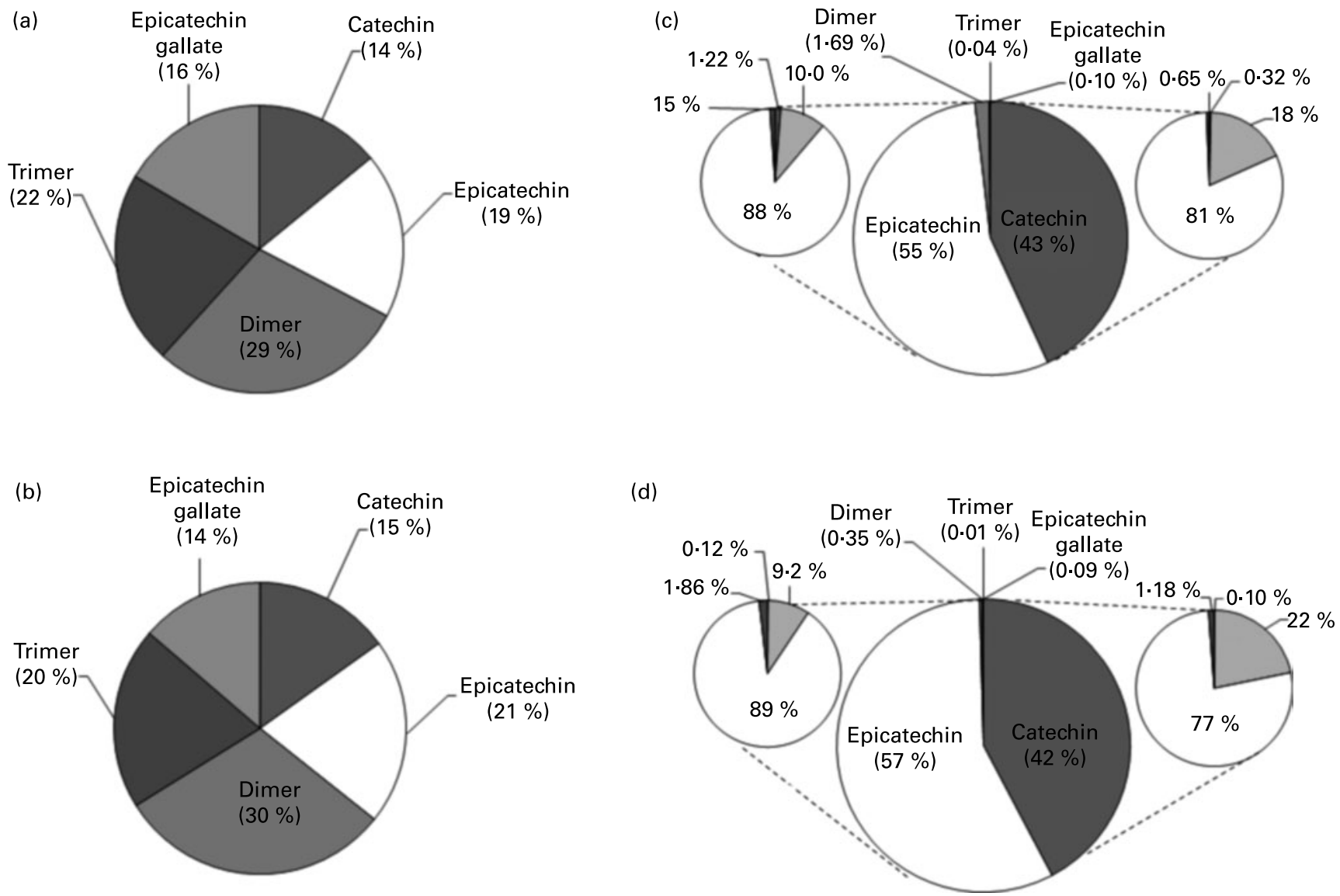

Fig. 4. ( $a$ and b) In vitro and in vivo grape seed procyanidin extract (GSPE). Percentage of the catechin, epicatechin, epicatechin gallate, dimer and trimer in the dialysed fraction (fraction found inside dialysis solution) of the duodenal mixture. (c and d) In vitro and in vivo GSPE + carbohydrate-rich food. Percentage of the catechin, epicatechin and their respective metabolites, epicatechin gallate, dimer and trimer in the postprandial rat plasma. 
effects of $\mathrm{GSPE}^{(2)}$. As there is some controversy about the bioavailability of dimeric and trimeric PC, we used a combination of in vitro and in vivo models in order to measure the bioavailability of dimer and trimer PC. The effects of the digestion process on their stability and the food matrix effect on their potential bioaccessibility were also evaluated.

The GSPE used as a source of PC in the present study contained a substantial amount of high molecular weight polymeric PC, dimer and trimer being the most abundant. After the simulated in vitro digestion, these PC oligomers were present in high concentration in all digestion fractions, showing an important stability under gastric and duodenal digestion conditions. The most important aspect is related to the increase in their concentration observed in the digestion mixtures after the two steps. This is probably related to the partial hydrolysis of the highly polymerised molecules or condensed tannins in the GSPE. However, the pharmacokinetic study revealed a limited absorption. The limitation in the diffusion across the epithelial cells could explain the nanomolar levels quantified in the postprandial rat plasma after the intake, at micromolar levels, of the oligomeric PC. The pharmacokinetic results shown in the present study agree with previous research that has shown the presence of some dimers (B2 and B5), detected at nanomolar levels, in the plasma of rats given cocoa extracts ${ }^{(35,36)}$. By contrast, in other studies, the dimer (B3) and trimer (C2) were not detected in the plasma of rats given purified compounds ${ }^{(37)}$. With regard to the metabolism, the major metabolites observed in rat plasma in the present study were glucuronidated forms of catechin and epicatechin, although there were also high levels of methylated-glucuronide forms. Similarly, a previous study by Spencer et al. ${ }^{(38)}$ reported that methylated-glucuronide forms were the most bioavailable forms in the small intestine.

When the GSPE extract was submitted to the in vitro digestion model, there were high concentrations of the monomers catechin and epicatechin and mainly the dimer and trimer oligomeric flavanols in the gastric fractions (chyme and pellet), showing an important stability under acid conditions. Similar results were observed by Spencer et al. ${ }^{(9)}$. The results of pancreatic digestion showed a lesser decrease in the concentration of PC with a high degree of polymerisation, because a slight decomposition of the oligomeric forms into monomers was seen after the duodenal digestion, being greater when the extract was digested with carbohydrate-rich food. Thus, PC were less stable under alkaline conditions $(\mathrm{pH}=6 \cdot 9)$ and in the presence of digestive enzymes than under acid conditions in the gastric step. After the evaluation of the stability of the PC under digestion conditions, the next phase was focused on the in vitro estimation of the potential bioaccessibility and potential absorption in the systemic circulation in biological systems. For this proposal, a dialysis phase was considered for the duodenal step, estimating the passive diffusion of the digested PC solubilised in the water phase (IN) (Fig. 1). Comparing the total concentration of $\mathrm{PC}$ in the duodenal mixture (IN and OUT) after the duodenal digestion with the total concentration in the digestion mixture after the gastric step, a substantial loss was observed. The losses in the PC content of the duodenal digestion mixture could be attributed to the large oligomers being held on the dialysis membrane, reducing the recovery of the $\mathrm{PC}$ to be analysed during the washing of the dialysis tub. Additionally, the large oligomers could reduce the membrane's surface. This problem was also reported by Bermúdez-Soto et al. ${ }^{(39)}$, who argued that the dialysis membrane as a means of estimating availability for absorption had some limitations. However, some authors have reported that $\mathrm{PC}$ are unstable under alkaline conditions ${ }^{(36,40,41)}$, and this instability also may explain the decrease of PC concentrations in the digestion mixture after pancreatic digestion. According to Rios et al. ${ }^{(19)}$, the stability of PC in the stomach and their very limited absorption in the small intestine suggest that they may influence digestion or the physiology of the gut through direct interactions with the gut mucosa and gut lumen solutes.

On the other hand, a decrease in the catechin:epicatechin ratio was observed during digestion in the in vitro model in the present study. The increase in epicatechin could be the consequence of the depolymerisation of GSPE, which is mainly composed of epicatechin units, into monomeric forms. However, in the study by Rios et al. ${ }^{(19)}$, no epimerisation was observed during the stomach digestion and oligomers to pentamers were stable during stomach transit. The results of the present study corresponding to the in vivo model revealed epicatechin and its metabolites as the predominant plasma monomer absorbed (Fig. 2). This fact has been also observed by other authors ${ }^{(42)}$ who showed in human subjects that after consuming a cocoa beverage containing equal amounts of epicatechin and catechin, the former was identified as the predominant plasma flavanol absorbed.

The pharmacokinetic study of the rat plasma revealed that the glucuronidated forms were the most abundant metabolite. These results agree with those in Vaidyanathan \& Walle ${ }^{(43)}$ who observed that rat liver microsomes efficiently glucuronidate epicatechin. Glucuronidation of catechin has been described to be more active in rat livers than in the intestine, in both in vitro ${ }^{(44)}$ and in vivo models ${ }^{(45)}$. Traces of glucuronidated and sulphated metabolites from monomers have also been detected in the lumen of gut from rats treated with $\mathrm{GSPE}^{(1)}$. One of the most important factors determining the metabolic fate of polyphenols in human subjects is the quantity ingested ${ }^{(46)}$. According to Hacket et al. ${ }^{(47)}$, when food polyphenols are administered at a pharmacological dose, they are found in free forms in the blood. By contrast, when the dose is decreased to only a few milligrams, the monomers were conjugated and no free forms were detected ${ }^{(18)}$. Related to this, in the present study, the free forms of catechin and epicatechin were found at very low concentrations in the plasma samples after the ingestion of GSPE. The present results may be coherent with the dose administered to the rats, because the dose used is an intermediate position between the pharmacological dose and typical dose of polyphenols present in rich foods.

The last objective in the present study was to evaluate the effect of carbohydrate-rich foods on the bioavailability of the GSPE. Relatively, little is known about the effect of food on the bioavailability of PC. Food within the gastrointestinal tract is known to markedly alter the oral absorption of many xenobiotic compounds ${ }^{(48)}$. In the present in vitro study, when the extract was ingested with carbohydrate-rich food, an enhanced uptake of the monomeric PC was observed. This result is in agreement with the results in Schramm et al. ${ }^{(26)}$, who reviewed that the effect observed with carbohydrate-rich foods (bread, sucrose and grapefruit juice) may 
have been mediated by a carbohydrate-specific effect on gastrointestinal physiology (e.g. motility and/or secretion) or a carbohydrate-specific enhancement of the activity of a yet unidentified carbohydrate-flavanol transporter.

In conclusion, dimer and trimer PC are absorbable in vivo, reaching maximum concentrations in the plasma as soon as $1 \mathrm{~h}$ after PC ingestion. Their low concentrations in the plasma are not the consequence of instability during the digestion process as the in vitro digestion model shows high levels of dimer and trimer after duodenal digestion. Moreover, absorption of dimer and trimer PC is repressed by the simultaneous presence of carbohydrate-rich food.

\section{Acknowledgements}

The present work was supported by the CENIT program from the Spanish Minister of Industry and by a consortium of companies led by La Morella Nuts S.A. (Reus, Catalonia, Spain) with the following companies: Shirota Functional Foods, S.L.; KRAFT; BTSA, Biotecnologías Aplicadas, S.L.; Selección Batallé, S.A.; Industrial Técnica Pecuaria, S.A.; Neuron BioPharma, S.A.; Grupo Leche Pascual, S.A.U.; Innaves, S.A. The present work was also supported by the Spanish Ministry of Education and Science financing the project AGL2008-00387. We would like to thank N. Llópiz, V. Grifoll and Y. Tobajas for their technical assistance, and the MITOFOOD network of which L. Arola is a member. The authors declare that no conflict of interest regarding the present article is present. Author contributions. M.-J. M., L. A. and C. B. had full access to all of the data in the study and take responsibility for the integrity of the data and the accuracy of the data analysis. M.-J. M., L. A. and C. B. did study concept and design. L. A. and J. V. did acquisition of data GSPE. A. S., M.-P. R. and A. M. did acquisition of data in vitro digestion. C. B., A. S. and A. M. did acquisition of data in vivo bioavailability. A. S., A. M., M.-J. M., C. B., L. A. and J. V. did analyses and interpretation of data. M.-J. M., L. A. and C. B. did drafting of the manuscript. A. S., A. M., M.-J. M., L. A. and C. B. did critical revision of the manuscript for important intellectual content. A. M., C. B. and J. V. did administrative, technical and logistic support.

\section{References}

1. Tsang C, Auger C, Mullen W, et al. (2005) The absorption, metabolism and excretion of flavan-3-ols and procyanidins following the ingestion of a grape seed extract by rats. Br J Nutr 94, 170-181.

2. Puiggròs F, Sala E, Vaque M, et al. (2009) In vivo, in vitro, and in silico studies of $\mathrm{Cu} / \mathrm{Zn}$-superoxide dismutase regulation by molecules in grape seed procyanidin extract. J Agric Food Chem 57, 3934-3942.

3. Del Bas JM, Ricketts ML, Baiges I, et al. (2008) Dietary procyanidins lower triglyceride levels signaling through the nuclear receptor small heterodimer partner. Mol Nutr Food Res 52, $1172-1181$.

4. Quesada H, Del Bas JM, Pajuelo D, et al. (2009) Grape seed proanthocyanidins correct dyslipidemia associated with a highfat diet in rats and repress genes controlling lipogenesis and VLDL assembling in liver. Int J Obes (Lond) 33, 1007-1012.

5. Pinent M, Bladé MC, Salvadó MJ, et al. (2005) Grape-seed derived procyanidins interfere with adipogenesis of 3T3-L1 cells at the onset of differentiation. Int $J$ Obes (Lond) 29 , 934-941.

6. Pinent M, Blay M, Bladé MC, et al. (2004) Grape seedderived procyanidins have an antihyperglycemic effect in streptozotocin-induced diabetic rats and insulinomimetic activity in insulin-sensitive cell lines. Endocrinology 145, 4985-4990.

7. Terra X, Montagut G, Bustos M, et al. (2009) Grape-seed procyanidins prevent low-grade inflammation by modulating cytokine expression in rats fed a high-fat diet. J Nutr Biochem 20, 210-218.

8. Montagut G, Baiges I, Valls J, et al. (2009) A trimer plus a dimer-gallate reproduce the bioactivity described for an extract of grape seed procyanidins. Food Chem 116, 265-270.

9. Spencer JPE, Chaudry F, Pannala AS, et al. (2000) Decomposition of cocoa procyanidins in the gastric milieu. Biochem Biophys Res Commun 272, 236-241.

10. Rein D, Lotito S \& Holt RR (2000) Epicatechin in human plasma: in vivo determination and effect of chocolate consumption on plasma oxidation status. J Nutr 130, Suppl. 8, 2109S-2114S.

11. Baba S, Osakabe N, Natsume M, et al. (2001) Absorption and urinary excretion of (-)-epicatechin after administration of different levels of cocoa powder or (-)-epicatechin in rats. J Agric Food Chem 49, 6050-6056.

12. Donovan JL, Crespy V, Oliveira M, et al. (2006) (+)-Catechin is more bioavailable than (-)-catechin: relevance to the bioavailability of catechin from cocoa. Free Radic Res 40, 1029-1034.

13. Wang J, Luo H, Wang P, et al. (2008) Validation of green tea polyphenol biomarkers in a phase II human intervention trial. Food Chem Toxicol 46, 232-240.

14. Yang CS, Chen L, Lee M, et al. (1998) Blood and urine levels of tea catechins after ingestion of different amounts of green tea by human volunteers. Cancer Epidemiol Biomarkers Prev 7 , 351-354.

15. Warden BA, Smith LS, Beecher GR, et al. (2001) Catechins are bioavailable in men and women drinking black tea throughout the day. J Nutr 131, 1731-1737.

16. Piskula MK \& Terao J (1998) Accumulation of (- )-epicatechin metabolites in rat plasma after oral administration and distribution of conjugation enzymes in rat tissues. J Nutr 128, $1172-1178$.

17. Donovan JL, Luthria DL, Stremple P, et al. (1999) Analysis of (+)-catechin, (-)-epicatechin and their $3^{\prime}$ - and $4^{\prime}$ - $O$-methylated analogs. A comparison of sensitive methods. J Chromatogr B Biomed Sci Appl 726, 277-283.

18. Bell JR, Donovan JL, Wong R, et al. (2000) (+)-Catechin in human plasma after ingestion of a single serving of reconstituted red wine. Am J Clin Nutr 71, 103-108.

19. Rios LY, Bennett RN, Lazarus SA, et al. (2002) Cocoa procyanidins are stable during gastric transit in humans. Am J Clin Nutr 76, 1106-1110.

20. Sano A, Yamakoshi J, Tokutake S, et al. (2003) Procyanidin B1 is detected in human serum after intake of proanthocyanidinrich grape seed extract. Biosci Biotechnol Biochem 67, $1140-1143$.

21. Scalbert A \& Williamson G (2000) Dietary intake and bioavailability of polyphenols. $J$ Nutr 130, Suppl. 8, 2073S-2085S.

22. Manach C, Scalbert A, Morand C, et al. (2004) Polyphenols: food sources and bioavailability. Am J Clin Nutr 79, 727-747.

23. Van Het Hof KH, Kivits GAA \& Weststrate JA (1998) Bioavailability of catechins from tea: the effect of milk. Eur $J$ Clin Nutr 52, 356-359.

24. Hollman PCH, Van Het Hof KH, Tijburg LBM, et al. (2001) Addition of milk does not affect the absorption of flavonols from tea in man. Free Radic Res 34, 297-300. 
25. Shishikura Y, Khokhar S \& Murray BS (2006) Effects of tea polyphenols on emulsification of olive oil in a small intestine model system. J Agric Food Chem 54, 1906-1913.

26. Schramm DD, Karim M, Schrader HR, et al. (2003) Food effects on the absorption and pharmacokinetics of cocoa flavanols. Life Sci 73, 857-869.

27. Laurent C, Besançon P \& Caporiccio B (2005) Ethanol and polyphenolic free wine matrix stimulate the differentiation of human intestinal Caco-2 cells. Influence of their association with a procyanidin-rich grape seed extract. J Agric Food Chem 53, 5541-5548.

28. Oomen AG, Hack A, Minekus M, et al. (2002) Comparison of five in vitro digestion models to study the bioaccessibility of soil contaminants. Environ Sci Technol 36, 3326-3334.

29. Bermúdez-Soto $\mathrm{M}$, Tomás-Barberán $\mathrm{F}$ \& García-Conesa $\mathrm{M}$ (2007) Stability of polyphenols in chokeberry (Aronia melanocarpa) subjected to in vitro gastric and pancreatic digestion. Food Chem 102, 865-874.

30. Afkhami F, Ouyang W, Chen H, et al. (2007) Impact of orally administered microcapsules on gastrointestinal microbial flora: in-vitro investigation using computer controlled dynamic human gastrointestinal model. Artif Cells Blood Substit Immobil Biotechnol 35, 359-375.

31. De Boever P, Deplancke B \& Verstraete W (2000) Fermentation by gut microbiota cultured in a simulator of the human intestinal microbial ecosystem is improved by supplementing a soygerm powder. J Nutr 130, 2599-2606.

32. Ortega N, Romero MP, Macià A, et al. (2008) Obtention and characterization of phenolic extracts from different cocoa sources. J Agric Food Chem 56, 9621-9627.

33. Serra A, Macià A, Romero MP, et al. (2009) Determination of procyanidins and their metabolites in plasma samples by improved liquid chromatography-tandem mass spectrometry. J Chromatogr B Anal Technol Biomed Life Sci 877, 1169-1176.

34. Ortega N, Reguant J, Romero MP, et al. (2009) Effect of fat content on the digestibility and bioaccessibility of cocoa polyphenol by an in vitro digestion model. J Agric Food Chem 57, 5743-5749.

35. Baba S, Osakabe $N$ \& Natsume $M$ (2002) Absorption and urinary excretion of procyanidin B2 [epicatechin-(4 $\beta-8)$-epicatechin] in rats. Free Radic Biol Med 33, 142-148.
36. Zhu QY, Holt RR, Lazarus SA, et al. (2002) Stability of the flavan-3-ols epicatechin and catechin and related dimeric procyanidins derived from cocoa. J Agric Food Chem 50, $1700-1705$.

37. Gonthier MP, Donovan JL, Texier O, et al. (2003) Metabolism of dietary procyanidins in rats. Free Radic Biol Med 35, 837-844.

38. Spencer JP (2003) Metabolism of tea flavonoids in the gastrointestinal tract. J Nutr 133, 3255S-3261S.

39. Bermúdez-Soto MJ, Tomás-Barberán $\mathrm{F}$ \& García-Conesa $\mathrm{M}$ (2007) Stability of polyphenols in chokeberry (Aronia melanocarpa) subjected to in vitro gastric and pancreatic digestion. Food Chem 102, 865-874.

40. Yoshino K, Suzuki M, Sasaki K, et al. (1999) Formation of antioxidants from (-)-epigallocatechin gallate in mild alkaline fluids, such as authentic intestinal juice and mouse plasma. J Nutr Biochem 10, 223-229.

41. Zhu QY, Zhang A, Tsang D, et al. (1997) Stability of green tea catechins. J Agric Food Chem 45, 4624-4628.

42. Holt RR, Lazarus SA, Cameron Sullards M, et al. (2002) Procyanidin dimer B2 [epicatechin- $(4 \beta-8)$-epicatechin] in human plasma after the consumption of a flavanol-rich cocoa. Am J Clin Nutr 76, 798-804.

43. Vaidyanathan JB \& Walle T (2002) Glucuronidation and sulfation of the tea flavonoid (-)-epicatechin by the human and rat enzymes. Drug Metab Dispos 30, 897-903.

44. Crespy V \& Williamson G (2004) A review of the health effects of green tea catechins in in vivo animal models. J Nutr 134, Suppl. 12, 3431S-3440S.

45. Lu H, Meng X, Li C, et al. (2003) Glucuronides of tea catechins: enzymology of biosynthesis and biological activities. Drug Metab Dispos 31, 452-461.

46. Spencer JPE, Abd El Mohsen MM, Minihane A, et al. (2008) Biomarkers of the intake of dietary polyphenols: strengths, limitations and application in nutrition research. Br J Nutr 99, 12-22.

47. Hackett AM, Griffiths LA, Broillet A, et al. (1983) The metabolism and excretion of $(+)-\left[{ }^{14} \mathrm{C}\right]$ cyanidanol-3 in man following oral administration. Xenobiotica 13, 279-286.

48. Charman WN, Porter CJH, Mithani S, et al. (1997) Physicochemical and physiological mechanisms for the effects of food on drug absorption: the role of lipids and pH. J Pharm Sci 86, $269-282$. 